\title{
Protograph-Based LDPC Code Design for Bit-Metric Decoding
}

\author{
Fabian Steiner, Georg Böcherer \\ Institute for Communications Engineering \\ Technische Universität München, Germany \\ Email: \{fabian.steiner, georg.boecherer\}@tum.de
}

\author{
Gianluigi Liva \\ Institute of Communication and Navigation of the \\ Deutsches Zentrum für Luft- und Raumfahrt (DLR) \\ 82234 Wessling, Germany \\ Email: Gianluigi.Liva@dlr.de
}

\begin{abstract}
A protograph-based low-density parity-check (LDPC) code design technique for bandwidth-efficient coded modulation is presented. The approach jointly optimizes the LDPC code node degrees and the mapping of the coded bits to the bit-interleaved coded modulation (BICM) bit-channels. For BICM with uniform input and for BICM with probabilistic shaping, binary-input symmetric-output surrogate channels are constructed and used for code design. The constructed codes perform as good as multi-edge type codes of Zhang and Kschischang (2013). For 64-ASK with probabilistic shaping, a blocklength 64800 code is constructed that operates within $0.69 \mathbf{d B}$ of $\frac{1}{2} \log _{2}(1+\mathbf{S N R})$ at a spectral efficiency of 4.25 bits/channel use and a frame error rate of $10^{-3}$.
\end{abstract}

Keywords-LDPC codes, protographs, surrogate channels, BICM, probabilistic shaping

\section{INTRODUCTION}

Bit-interleaved coded modulation (BICM) combines high order modulation with binary error correcting codes [1], [2]. This makes BICM attractive for practical application and BICM is widely used in standards, e.g., in DVB-T2/S2/C2. At a $\mathrm{BICM}$ receiver, bit-metric decoding (BMD) is used [3, Sec. II]. Achievable rates for BMD were investigated for uniformly distributed inputs in [3] and for non-uniformly distributed bits in [4]. These results were generalized to non-uniformly distributed input symbols in [5] and [6].

When designing low-density parity-check (LDPC) codes for BICM, the key challenge is to take into account the unequal error protection of the LDPC coded bits and the BICM bitchannels that are different for different bit-levels [7, Sec. VI]. A first approach is to take an existing LDPC code and to optimize the mapping of the coded bits to the BICM bit-levels. This was done, e.g., in [8]-[11]. A more fundamental approach is to directly incorporate the different bit-channels in the code design. This is done in [12], where the authors use multiedge type (MET) codes [13] to parameterize the different bitchannels. They then extend the extrinsic information transfer charts (EXIT) [14] to multiple dimensions to design codes for quadrature amplitude modulation with 16 signal points (16QAM). Since 16-QAM can be constructed as the Cartesian product of two four point amplitude-shift keying (4-ASK) constellations, two different bit-channels are apparent. For constellations with more than two different bit-channels, the authors of [12] observe long runtimes of their multidimensional EXIT approach. Therefore, they suggest a high-order extension based on nesting, i.e., starting from $m=2$, they successively extend their codes from $m$ to $m+1$ bit-levels by optimizing in each step only the additional bit-level.

In this work, we follow [12] and jointly optimize the code structure and the mapping of the coded bits to the BICM bitlevels. However, we propose a protograph-based design [15] and use protograph EXIT (PEXIT) analysis [16] to determine the ensemble iterative convergence threshold by accounting for the different bit-channels associated with the protograph variable nodes. The protograph ensemble is optimized with respect to the threshold by differential evolution [17]. A design of protographs for coded modulation was first introduced in [18], which relies on a variable degree matched mapping (VDMM). More specifically, in [18] each bit-level is associated to a specific protograph variable node following the waterfilling approach (i.e., assigning the most protected coded bits to the bit-levels with highest bit-channel capacities). Recently, a protograph-based coded modulation scheme was introduced in [19] by performing a one-to-one mapping between the constellation symbols and the codeword symbols of a nonbinary protograph LDPC code. This requires the constellation order to match the field order on which the LDPC code is constructed. To the best of our knowledge, none of the abovementioned approaches leads to a joint binary LDPC protograph ensemble and bit-mapping optimization.

For the PEXIT analysis, we represent each bit-channel by a surrogate. Our surrogates reflect both the BICM bitchannels and the input distribution. We optimize codes both for uniformly distributed inputs and for the probabilistic shaping strategy proposed in [5], [7]. Our optimized codes show a finite length performance that is as good as the one presented by Zhang and Kschischang [12]. Moreover, our design approach can be applied to very large constellations. For 64-ASK with probabilistic shaping, our blocklength 64800 code operates within $0.69 \mathrm{~dB}$ of $\frac{1}{2} \log _{2}(1+\mathrm{SNR})$ at a spectral efficiency of $4.25 \mathrm{bits} / \mathrm{channel}$ use and a frame error rate of $10^{-3}$.

This paper is organized as follows. In Sec. II, we review bit-metric decoding. We present our code design approach in Sec. III. In Sec. IV and Sec. V, we discuss the performance of our codes for uniform and shaped inputs, respectively.

\section{Bit-Metric DeCODING}

Consider the discrete time additive white Gaussian noise (AWGN) channel

$$
Y=\Delta X+Z
$$




\section{0}

Figure 1. 8-ASK constellation with BRGC [20] labeling.

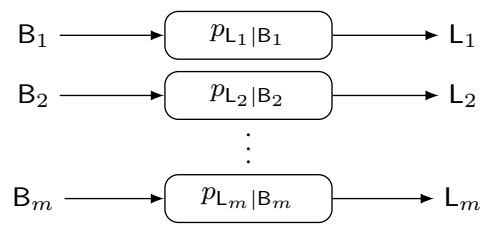

Figure 2. $m$ parallel bit-channels that are different for different bit-levels.

where the noise term $Z$ is zero mean, unit variance Gaussian, and where the input $X$ is distributed on the normalized $2^{m}$. ASK constellation

$$
\mathcal{X}=\left\{ \pm 1, \pm 2, \ldots, \pm\left(2^{m}-1\right)\right\} .
$$

The constellation spacing $\Delta$ controls the average power $\mathrm{E}\left[|\Delta \mathrm{X}|^{2}\right]$, where $\mathrm{E}[\cdot]$ denotes expectation. The signal-to-noise ratio (SNR) is $\mathrm{SNR}=\mathrm{E}\left[|\Delta \mathrm{X}|^{2}\right] / 1$. Each signal point $x \in \mathcal{X}$ is labeled by $m$ bits $\mathbf{B}=\left(\mathrm{B}_{1}, \mathrm{~B}_{2}, \ldots, \mathrm{B}_{m}\right)$. The $\mathrm{B}_{i}$ represent the bit-levels. Throughout this work, we label by the binary reflected Gray code (BRGC) [20], e.g., see Fig. 1. Let $p_{\mathrm{Y} \mid \mathbf{B}}$ be the memoryless channel with random input $\mathrm{B}$ and output $\mathrm{Y}$. The bit-metric of bit-level $i$ is

$$
L_{i}=\log \frac{P_{\mathrm{B}_{i} \mid \mathrm{Y}}(0 \mid y)}{P_{\mathrm{B}_{i} \mid \mathrm{Y}}(1 \mid y)},
$$

where $P_{\mathrm{B}_{i} \mid \mathrm{Y}}$ can be calculated from the joint distribution

$$
P_{\mathrm{B}_{i}}\left(b_{i}\right) p_{Y \mid \mathrm{B}_{i}}\left(y \mid b_{i}\right)=\sum_{\boldsymbol{a} \in\{0,1\}^{m}: a_{i}=b_{i}} p_{\mathrm{Y} \mid \mathbf{B}}(y \mid \boldsymbol{a}) P_{\mathbf{B}}(\boldsymbol{a}) .
$$

A bit-metric decoder uses $L_{1}, L_{2}, \ldots, L_{m}$ to estimate the transmitted data. For the decoder, the channel appears as $m$ parallel bit-channels, see Fig. 2. Bit-metric decoding can achieve the rate [6, Theorem 1]

$$
R_{\mathrm{BMD}}=\mathbb{H}(\mathbf{B})-\sum_{i=1}^{m} \mathbb{H}\left(\mathrm{B}_{i} \mid \mathrm{L}_{i}\right)
$$

where $\mathbb{H}(\cdot)$ denotes entropy.

Remark 1. If the bits $\mathrm{B}_{1}, \mathrm{~B}_{2}, \ldots, \mathrm{B}_{m}$ are independent, then (3) can be written as [3], [4]

$$
R_{\mathrm{BMD}}=\sum_{i=1}^{m} \mathbb{I}\left(\mathrm{B}_{i} ; \mathrm{L}_{i}\right)
$$

where $\mathbb{I}(\cdot ; \cdot)$ denotes mutual information.

We use the achievable rate as defined in (3), since we will use non-uniformly distributed inputs in Sec. V, which also cause a stochastical dependence of some of the bit-levels.

\section{LDPC CODE DESIGN}

LDPC code ensembles are usually characterized by the degree profiles of the variable and check nodes of the Tanner graph representation of the sparse binary parity-check matrix $\boldsymbol{H} \in \mathbb{F}_{2}^{(n-k) \times n}[21$, Section 3.3]. For instance,

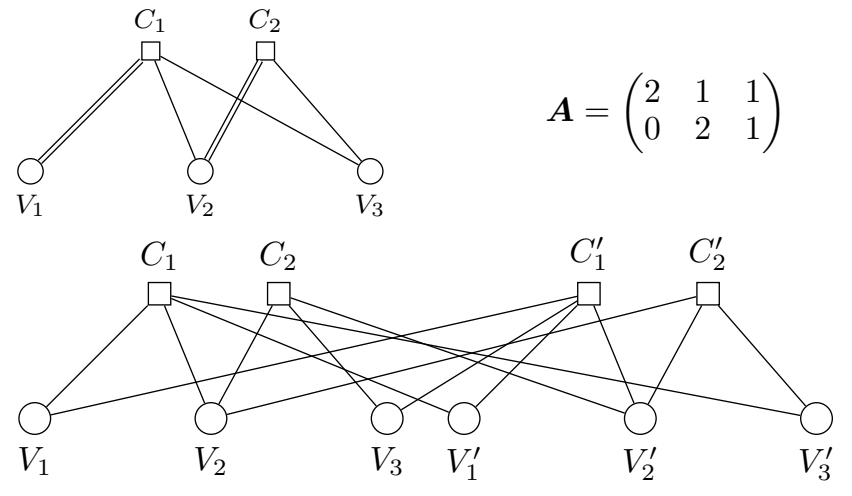

Figure 3. Above, the Tanner graph of basematrix $\boldsymbol{A}$ with $e=2$ and $f=3$ is displayed. Below, an example lifting with $Q=2$ instances of the protograph is shown.

$\lambda(x)=\sum_{d=1}^{d_{v}} \lambda_{d} x^{d-1}$ and $\rho(x)=\sum_{d=1}^{d_{c}} \rho_{d} x^{d-1}$ are the edgeperspective variable and check node degree polynomials with maximum degree $d_{v}$ and $d_{c}$, respectively. However, the degree profiles do not characterize the mapping of variable nodes to different bit-channels. In the following, we use protographs to incorporate the bit-mapping in our threshold analysis.

\section{A. Protograph-Based LDPC Codes}

Starting from a small bipartite graph represented via its basematrix $\boldsymbol{A}=\left[a_{l k}\right]$ of size $e \times f$, one applies a copy-andpermute operation (also known as lifting) to create $Q$ instances of the small graph and then permutes the edges so that the local edge connectivity remains the same. The $Q$ replicas of variable node $V_{k}, k \in\{1, \ldots, f\}$ must be connected only to replicas of the neighbors of $V_{k}$ while maintaining the original degrees for that specific edge. The resulting bipartite graph representing the final parity-check matrix $\boldsymbol{H}$ possesses $n=Q \cdot f$ variable nodes and $n-k=Q \cdot e$ check nodes. Parallel edges are allowed, but must be resolved during the copy-and-permute procedure. An example protograph with the corresponding basematrix and an example lifting for $Q=2$ are shown in Figure 3.

\section{B. Surrogate Channels}

Optimizing LDPC codes directly for the $m$ bit-channels in Fig. 2 is difficult, as they are usually not output-symmetric and do not possess uniform input in the case of probabilistic shaping. We instead optimize for a surrogate channel. The optimized code works well on the original channel if the surrogate channel preserves some characteristics of the original channel and if the code is universal in the sense that its performance depends mainly on these characteristics. We follow [7, Sec. VIII] and use the rate backoff as the key characteristic, i.e., if $R^{*}$ is the achievable rate of the original channel and $R$ is the actual transmission rate of the code, we assume that the decoding error probability depends on the rate backoff

$$
R^{*}-R \text {. }
$$

We design codes for a surrogate channel with the same rate backoff. Let $\tilde{R}$ be the transmission rate and $\tilde{R}^{*}$ the achievable rate of the surrogate channel; we require

$$
\tilde{R}^{*}-\tilde{R} \stackrel{!}{=} R^{*}-R .
$$




$$
\begin{aligned}
I_{E_{V_{k} \rightarrow C_{l}}} & =\mathrm{J}\left(\sqrt{\left.\sum_{\substack{l^{\prime}=1 \\
l^{\prime} \neq l}}^{e} a_{l^{\prime} k} \cdot \mathrm{J}^{-1}\left(I_{E_{C_{l^{\prime}} \rightarrow V_{k}}}\right)^{2}+\left(a_{l k}-1\right) \cdot \mathrm{J}^{-1}\left(I_{E_{C_{l} \rightarrow V_{k}}}\right)^{2}+\sigma_{\mathrm{ch}_{k}}^{2}\right)}\right. \\
I_{E_{C_{l} \rightarrow V_{k}}} & =1-\mathrm{J}\left(\sqrt{\sum_{\substack{k^{\prime}=1 \\
k^{\prime} \neq k}}^{f} a_{l k^{\prime}} \mathrm{J}^{-1}\left(1-I_{E_{V_{k^{\prime}} \rightarrow C_{l}}}\right)^{2}+\left(a_{l k}-1\right) \cdot \mathrm{J}^{-1}\left(1-I_{E_{V_{k}} \rightarrow C_{l}}\right)^{2}}\right) \\
I_{\mathrm{APP}_{k}} & =\mathrm{J}\left(\sqrt{\sum_{l^{\prime}=1}^{f} a_{l^{\prime} k} \cdot \mathrm{J}^{-1}\left(I_{E_{C_{l^{\prime}} \rightarrow V_{k}}}\right)^{2}+\sigma_{\mathrm{ch}_{k}}^{2}}\right) .
\end{aligned}
$$

Suppose our code rate is $c$ so that $(1-c) m$ bits per channel use consist of redundancy bits on average. Thus, the transmission rate is

$$
\mathbb{H}(\mathbf{B})-(1-c) m
$$

For instance, for uniformly distributed inputs, the transmission rate is $R=\mathrm{cm}$. By (3) and (7), the rate backoff is

$$
R^{*}-R=(1-c) m-\sum_{i=1}^{m} \mathbb{H}\left(\mathrm{B}_{i} \mid \mathrm{L}_{i}\right)
$$

Note that the term $(1-c) m$ does not depend on the channel. Thus, we operate the surrogate channel at the same rate backoff if

$$
\sum_{i=1}^{m} \mathbb{H}\left(\mathrm{B}_{i} \mid \mathrm{L}_{i}\right) \stackrel{!}{=} \sum_{i=1}^{m} \mathbb{H}\left(\tilde{\mathrm{B}}_{i} \mid \tilde{\mathrm{Y}}_{i}\right)
$$

Since we want to account for the different bit-channels, we restrict our surrogate channel further by requiring equality for the individual conditional entropies in (8) for each bit-level. We use biAWGN channels with uniform input as surrogate channel, i.e., we have

$$
\tilde{\mathrm{Y}}_{i}=x_{\tilde{\mathrm{B}}_{i}}+\mathrm{Z}_{i}
$$

where the $\tilde{\mathrm{B}}_{i}$ are binary and uniformly distributed, $x_{0}=1$, $x_{1}=-1$, and where $Z_{i}$ is zero mean Gaussian with variance $\sigma_{B_{i}}^{2}$ where

$$
\sigma_{B_{i}}^{2}: \quad \mathbb{H}\left(\mathrm{B}_{i} \mid \mathrm{L}_{i}\right) \stackrel{!}{=} \mathbb{H}\left(\tilde{\mathrm{B}}_{i} \mid \tilde{\mathrm{Y}}_{i}\right)
$$

\section{PEXIT Analysis}

The analysis of the asymptotic decoding threshold can be done by PEXIT analysis [16]. For EXIT chart analysis, the J function is widely used. The value $\mathrm{J}\left(\sigma_{\mathrm{ch}}\right)$ hereby represents the capacity of the biAWGNC with variance $\sigma^{2}$, where $\sigma_{\mathrm{ch}}^{2}=$ $\frac{4}{\sigma^{2}}$ :

$$
\mathrm{J}\left(\sigma_{\mathrm{ch}}\right)=1-\int_{-\infty}^{\infty} \frac{1}{\sqrt{2 \pi \sigma_{\mathrm{ch}}^{2}}} \mathrm{e}^{-\frac{\left(\ell-\sigma_{\mathrm{ch}}^{2} / 2\right)^{2}}{2 \sigma_{\mathrm{ch}}^{2}}} \log _{2}\left(1+\mathrm{e}^{-\ell}\right) \mathrm{d} \ell .
$$

PEXIT analysis requires tracking the extrinsic mutual information values $I_{E}$ per $\left(V_{k}, C_{l}\right)$ pair to accommodate for the edge connections imposed by the protograph basematrix and the (possibly different) reliability of the coded bits $V_{k}$. The PEXIT algorithm is summarized as follows:
1) Initialize each $\sigma_{\mathrm{ch}_{k}}, k \in\{1, \ldots, f\}$ with a corresponding $\sigma_{B_{i}}, i \in\{1, \ldots, m\}$ (9) originating from the biAWGNC surrogates. For all tuples $\left(V_{k}, C_{l}\right), k \in$ $\{1, \ldots, f\}, l \in\{1, \ldots, e\}$, set the a priori mutual information values $I_{E_{C_{l} \rightarrow V_{k}}}=0$.

2) Perform variable to check node update (4) for all tuples $\left(V_{k}, C_{l}\right)$.

3) Perform check to variable node update (5) for all tuples $\left(V_{k}, C_{l}\right)$.

4) Calculate $I_{\mathrm{APP}_{k}}, k \in\{1, \ldots, f\}$ (6).

5) Stop if all $I_{\mathrm{APP}_{k}}=1$, otherwise go to 2).

\section{CODE DESIGN FOR UNIFORM INPUTS}

\section{A. Optimization Procedure for Protograph Ensembles}

We impose restrictions on the dimension of the basematrix $\boldsymbol{A} \in\{0,1, \ldots, S\}^{e \times f}$. The parameters $e$ and $f$ determine how many different variable degrees per bit-channel can occur and are chosen to represent the desired code rate $c=\frac{f-e}{f}$. The maximum number of parallel edges $S$ is crucial for both the performance of the code and the success of the optimization procedure. The product $e \cdot S$ describes the maximum variable node degree in the final code and thereby determines the size of the optimization search space. The optimization of the basematrices is performed by differential evolution (DE) [22].

\section{B. Numerical Results}

In order to compare our code design approach to the setting of Zhang and Kschischang [12], we design codes of rates $1 / 2$ and $3 / 4$ for 4 -ASK constellations with uniform inputs. The optimized protographs are depicted in Table I. We discuss the rate $1 / 2$ code in more detail. The coded bits are transmitted over two different bit-channels. For each bit-channel, we allow 3 (possibly different) variable degrees so that always 3 variable nodes in the protograph are assigned to the same surrogate channel: $\sigma_{\mathrm{ch}_{1}}^{2}=\sigma_{\mathrm{ch}_{2}}^{2}=\sigma_{\mathrm{ch}_{3}}^{2}=4 / \sigma_{B_{2}}^{2}, \sigma_{\mathrm{ch}_{4}}^{2}=\sigma_{\mathrm{ch}_{5}}^{2}=$ $\sigma_{\mathrm{ch}_{6}}^{2}=4 / \sigma_{B_{3}}^{2}$. Once the optimized basematrices have been found, we construct quasi-cyclic parity-check matrices with blocklengths $n=16200$. We simulate the constructed codes using 100 decoding iterations. The bit error rates (BER) and frame error rates (FER) in Fig. 4 and 5 show that the finite length performance of our codes is equal to or slightly better than the codes in [12]. The decoding thresholds given in Table I are obtained by PEXIT analysis for the surrogate channels. 
Table I. OPTIMIZED PROTOGRAPHS AND DESIGN PARAMETERS

Constellation (code rate) Basematrix

PEXIT Decoding $\quad$ Gap to $R_{\mathrm{BMD}}$ (3) Threshold

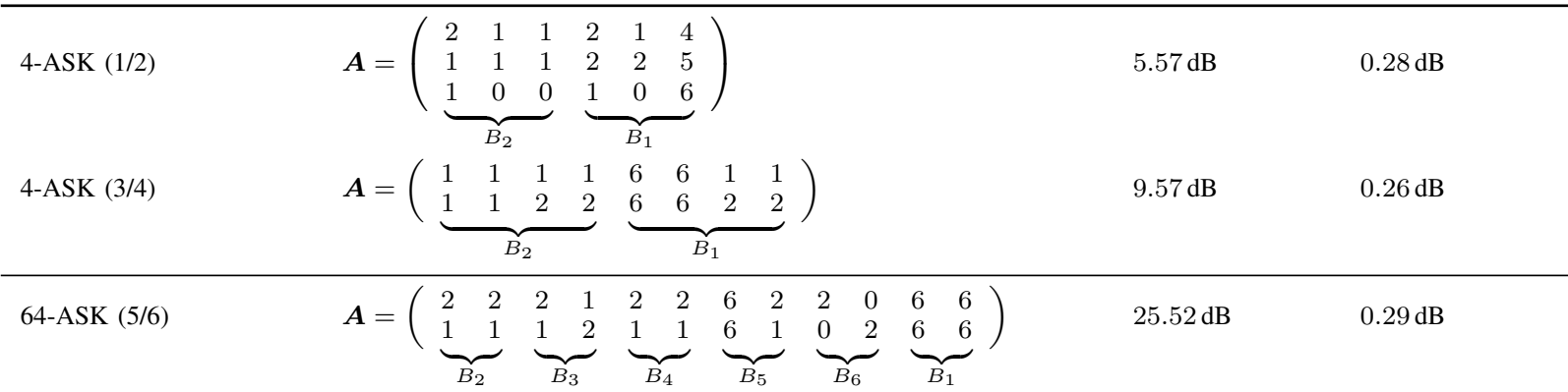

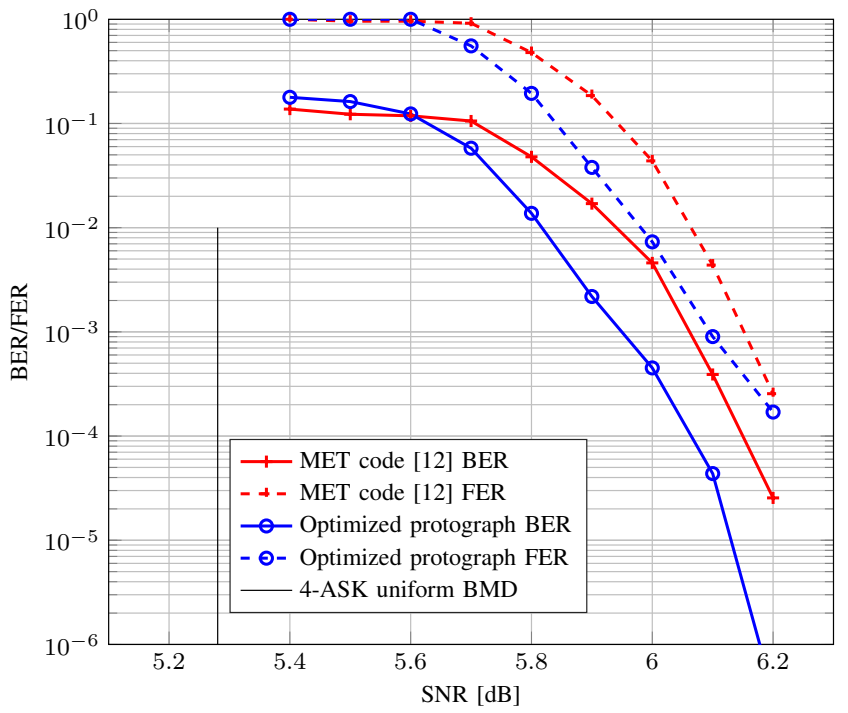

Figure 4. 4-ASK: Performance of a rate $1 / 2$ optimized protograph code compared to the rate $1 / 2$ MET code in [12].

\section{COdE DESIGN FOR Shaped INPUTS}

We briefly outline how our design procedure can be used to design LDPC codes for the probabilistic shaping scheme proposed in [5].

\section{A. Probabilistic Shaping}

Fig. 6 illustrates the shaping scheme. The key observation is that the capacity-achieving distribution of ASK constellations in Gaussian noise is symmetric around the origin. Consequently, the capacity-achieving distribution induces a distribution $P_{\mathrm{B}_{1} \mathrm{~B}_{2} \cdots \mathrm{B}_{m}}$ on the BRGC labeling with the following properties:

- Bit-level $B_{1}$ is uniformly distributed (bit-level $B_{1}$ decides on the sign of the transmitted constellation point, see Fig. 1).

- Bit-levels $\left(\mathrm{B}_{2} \cdots \mathrm{B}_{m}\right)$ and bit-level $\mathrm{B}_{1}$ are independent. Bit-levels $B_{2}, \ldots, B_{m}$ are correlated.

This suggests to mimic the capacity-achieving distribution in the following way: First, generate bit-levels $B_{2} \cdots B_{m}$ according to $P_{\mathrm{B}_{2} \cdots \mathrm{B}_{m}}$ by using a distribution matcher (see

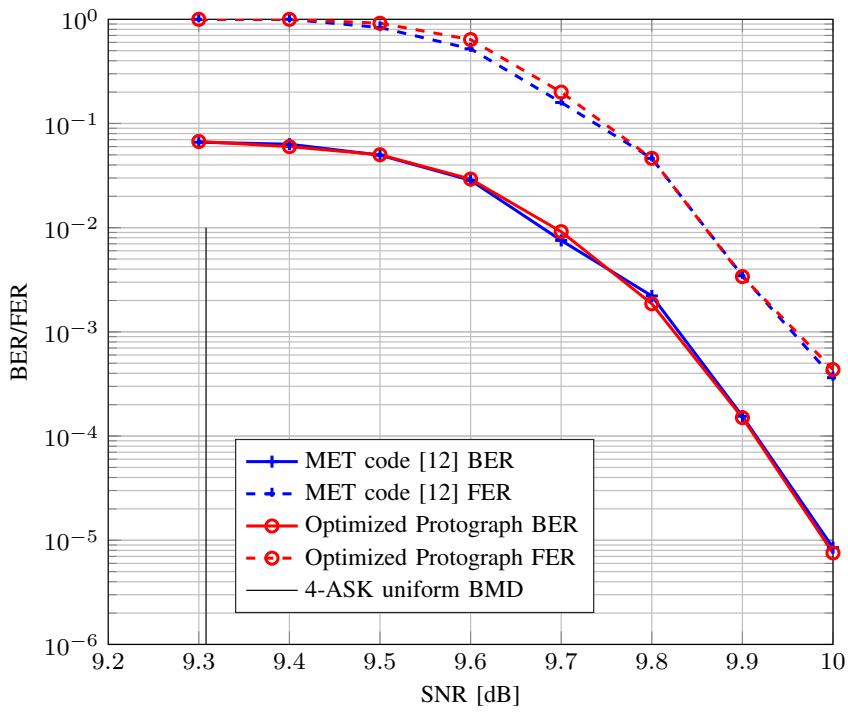

Figure 5. 4-ASK: Performance of a rate $3 / 4$ optimized protograph code compared to the rate $3 / 4$ MET code in [12]

Fig. 6). We use the distribution matcher proposed in [23]. Then encode by a systematic encoder of rate $(m-1) / m$ that copies the bits $\mathrm{B}_{2} \cdots \mathrm{B}_{m}$ to its output and leaves their distribution un-changed. The encoder appends check bits $B_{1}$ that are approximately uniformly distributed because each check bit is a modulo two sum of many information bits [24, Sec. 7.1]. The signal point $x_{\mathrm{B}_{1} \mathrm{~B}_{2} \cdots \mathrm{B}_{m}}$ selected by the bit-mapper then has approximately the capacity-achieving distribution.

\section{B. Surrogate Channels}

As discussed before, the channel appears to the receiver as a set of $m$ parallel binary input channels, see Fig. 2. The non-uniform distribution $P_{\mathrm{B}_{2} \cdots \mathrm{B}_{m}}$ influences each channel law $p_{\mathrm{L}_{i} \mid \mathrm{B}_{i}}$ via (2). Consequently, it also influences the conditional entropies $\mathbb{H}\left(B_{i} \mid L_{i}\right)$ in our surrogate channels (9) that we use for code optimization.

\section{Numerical Results}

We optimize codes for 64-ASK with probabilistic shaping. To obtain a close-to-optimal input distribution, we use the approach of [25], [7, Sec. 3C] with a sampled Gaussian (Maxwell-Boltzmann) distribution. We maximize the resulting 


$$
\mathrm{U}^{k_{\mathrm{d}}} \rightarrow \text { Matcher } \rightarrow\left(\mathrm{B}_{2} \cdots \mathrm{B}_{m}\right)^{n_{\mathrm{c}}} \rightarrow \text { LDPC Encoder } \rightarrow\left(\mathrm{B}_{2} \cdots \mathrm{B}_{m}\right)^{n_{\mathrm{c}}} \mathrm{B}_{1}^{n_{\mathrm{c}}} \rightarrow \text { Bit-Mapper } \rightarrow \mathrm{X}^{n_{\mathrm{c}}}
$$

Figure 6. Probabilistic shaping as proposed in [5], [7]. Independent uniformly distributed data bits $\mathrm{U}^{k_{\mathrm{d}}}=\mathrm{U}_{1} \mathrm{U}_{2} \cdots \mathrm{U}_{k_{\mathrm{d}}}$ are matched to $n_{\mathrm{c}}$ strings $\left(\mathrm{B}_{2} \mathrm{~B}_{3} \cdots \mathrm{B}_{m}\right)_{i}$, $i=1,2, \ldots, n_{\mathrm{c}}$ that are distributed according to $P_{\mathrm{B}_{2} \cdots \mathrm{B}_{m}}$. The systematic LDPC encoder appends the $n_{\mathrm{c}}$ check bits $\mathrm{B}_{1}^{n_{\mathrm{c}}}=\mathrm{B}_{11} \mathrm{~B}_{12} \cdots \mathrm{B}_{1 n_{\mathrm{c}}}$. The bit mapper maps this bit stream to signal points according to the BRGC labeling. The overall rate is $k_{\mathrm{d}} / n_{\mathrm{c}}$ [bits/channel use] and the rate of the LDPC code is $(m-1) n_{\mathrm{c}} /\left(m n_{\mathrm{c}}\right)=(m-1) / m$. At the receiver, a bit-metric decoder calculates an estimate of $\left(\mathrm{B}_{2} \cdots \mathrm{B}_{m}\right)^{n_{\mathrm{c}}} \mathrm{B}_{1}^{n_{\mathrm{c}}}$. A data estimate $\hat{\mathrm{U}}^{k_{\mathrm{d}}}=\hat{\mathbf{U}}_{1} \hat{\mathrm{U}}_{2} \cdots \hat{\mathrm{U}}_{k_{\mathrm{d}}}$ is obtained by passing the estimate of $\left(\mathrm{B}_{2} \cdots \mathrm{B}_{m}\right)^{n_{\mathrm{c}}}$ through a dematcher. For the matcher and the dematcher, we use the implementation proposed in [23].

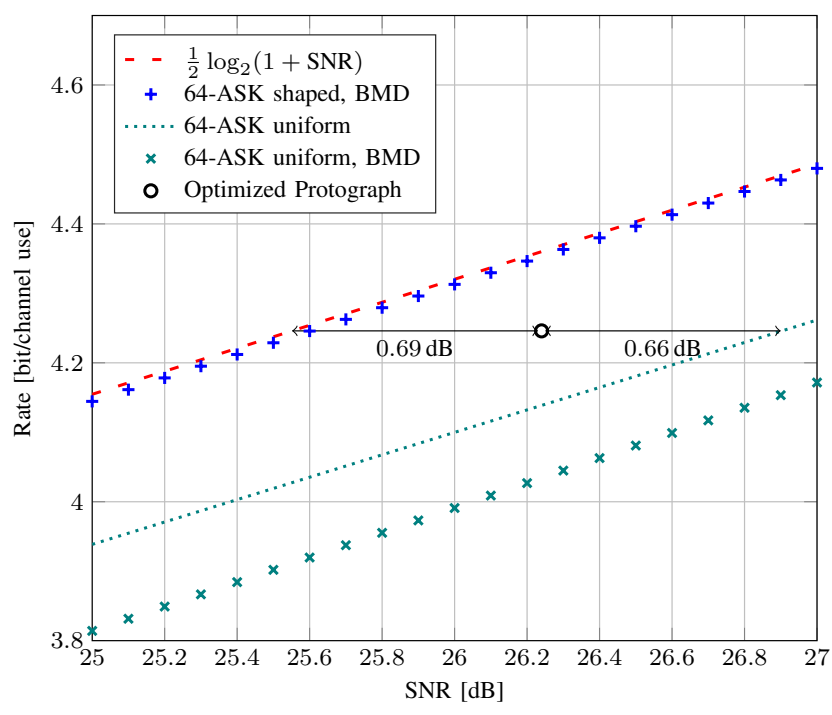

Figure 7. 64-ASK: The operating point of an optimized protograph code of blocklength $n=64800$ and FER $=10^{-3}$. For comparison, AWGN capacity and power-rate curves are displayed.

BMD rate (3) over the constellation spacing $\Delta$ in (1). The results are shown in Fig. 7. Our optimized protograph code of blocklength $n=64800$ shows a gap of $0.69 \mathrm{~dB}$ to AWGN capacity at $4.25 \mathrm{bits} / \mathrm{channel}$ use for $\mathrm{FER}=10^{-3}$. Our operating point is $0.66 \mathrm{~dB}$ more energy efficient than uniform 64-ASK capacity and $1.17 \mathrm{~dB}$ more energy efficient than uniform 64ASK capacity with bit-metric decoding.

\section{CONCLUSION}

We proposed a protograph-based LDPC code design approach for bandwidth-efficient coded modulation that is suitable both for uniform and shaped inputs. The different bitchannels are replaced by biAWGN surrogates so that PEXIT and differential evolution give ensembles with good decoding thresholds. The performance of the new codes for uniform inputs are as good as the best codes in literature. For shaped inputs, the new codes operate close to $\frac{1}{2} \log _{2}(1+\mathrm{SNR})$. Future research should investigate the influence of the surrogates on the code performance by employing a full-fledged density evolution for protographs. Furthermore, precoded protographs should be considered to improve the threshold without increasing the variable node degrees.

\section{REFERENCES}

[1] E. Zehavi, "8-PSK trellis codes for a Rayleigh channel," IEEE Trans. Commun., vol. 40, no. 5, pp. 873-884, May 1992.

[2] G. Caire, G. Taricco, and E. Biglieri, "Bit-interleaved coded modulation," IEEE Trans. Inf. Theory, vol. 44, no. 3, pp. 927-946, 1998.
[3] A. Martinez, A. Guillen i Fabregas, G. Caire, and F. Willems, "Bitinterleaved coded modulation revisited: A mismatched decoding perspective," IEEE Trans. Inf. Theory, vol. 55, no. 6, pp. 2756-2765, June 2009.

[4] A. Guillén i Fàbregas and A. Martinez, "Bit-interleaved coded modulation with shaping," in IEEE Inf. Theory Workshop (ITW), 2010.

[5] G. Böcherer, "Probabilistic signal shaping for bit-metric decoding," in Proc. IEEE Int. Symp. Inf. Theory (ISIT), June 2014, pp. 431-435.

[6] _ , "Achievable rates for shaped bit-metric decoding," arXiv preprint, no. 1410.8075, 2014. [Online]. Available: http://arxiv.org/abs/1410.8075

[7] G. Böcherer, P. Schulte, and F. Steiner, "Bandwidth efficient and ratematched low-density parity-check coded modulation," arXiv preprint, 2015. [Online]. Available: http://arxiv.org/abs/1502.02733

[8] Y. Li and W. Ryan, "Bit-reliability mapping in LDPC-coded modulation systems," IEEE Commun. Lett., vol. 9, no. 1, pp. 1-3, Jan 2005.

[9] J. Lei and W. Gao, "Matching graph connectivity of LDPC codes to high-order modulation by bit interleaving," in Proc. Allerton Conf. Commun., Contr., Comput., Sept 2008, pp. 1059-1064.

[10] C. Häger, A. Graell i Amat, A. Alvarado, F. Brännström, and E. Agrell, "Optimized bit mappings for spatially coupled LDPC codes over parallel binary erasure channels," in Proc. IEEE Int. Conf. Commun. (ICC), Jun. 2014, pp. 2064-2069.

[11] C. Häger, A. Graell i Amat, F. Brännström, A. Alvarado, and E. Agrell, "Improving soft FEC performance for higher-order modulations via optimized bit channel mappings," Opt. Express, vol. 22, no. 12, pp. 14544-14558, Jun 2014.

[12] L. Zhang and F. Kschischang, "Multi-edge-type low-density paritycheck codes for bandwidth-efficient modulation," IEEE Trans. Commun., vol. 61, no. 1, pp. 43-52, January 2013.

[13] T. Richardson and R. Urbanke, "Multi-edge type LDPC codes," Workshop honoring Prof. Bob McEliece on his 60th birthday, California Institute of Technology, Pasadena, California, pp. 24-25, 2002.

[14] S. ten Brink, "Convergence of iterative decoding," Electron. Lett., vol. 35, no. 10, pp. 806-808, 1999.

[15] J. Thorpe, "Low-density parity-check (LDPC) codes constructed from protographs," IPN progress report, vol. 42, no. 154, pp. 42-154, 2003.

[16] G. Liva and M. Chiani, "Protograph LDPC code design based on EXIT analysis," in IEEE Global Telecommun. Conf. (GLOBECOM), 2007, pp. $3250-3254$.

[17] R. Storn and K. Price, "Differential evolution-a simple and efficient heuristic for global optimization over continuous spaces," Journal of global optimization, vol. 11, no. 4, pp. 341-359, 1997.

[18] D. Divsalar and C. Jones, "Protograph based low error floor LDPC coded modulation," in IEEE Mil. Commun. Conf. (MILCOM), Oct 2005, pp. $378-385$ Vol. 1.

[19] A. Marinoni, P. Savazzi, and R. Wesel, "Protograph-based q-ary LDPC codes for higher-order modulation," in Proc. Int. Symp. Turbo Codes and Iterative Inf. Process. (ISTC), Sep. 2010, pp. 68-72.

[20] F. Gray, "Pulse code communication," Mar. 17 1953, US Patent $2,632,058$.

[21] T. Richardson and R. Urbanke, Modern Coding Theory. Cambridge Univ. Press, 2008.

[22] T. J. Richardson, M. A. Shokrollahi, and R. L. Urbanke, "Design of capacity-approaching irregular low-density parity-check codes," IEEE Trans. Inf. Theory, vol. 47, no. 2, pp. 619-637, 2001.

[23] P. Schulte and G. Böcherer, "Constant composition distribution matching," arXiv preprint, 2015. [Online]. Available: http://arxiv.org/ abs $/ 1503.05133$

[24] G. Böcherer, "Capacity-achieving probabilistic shaping for noisy and noiseless channels," Ph.D. dissertation, RWTH Aachen University, 2012

[25] F. Kschischang and S. Pasupathy, "Optimal nonuniform signaling for Gaussian channels," IEEE Trans. Inf. Theory, vol. 39, no. 3, pp. $913-$ 929, May 1993. 